\title{
Adjustable Flange Surgical Tracheostomy Audit: Best Practice
}

Guidelines

A Darr, S Azam, S Ahanger, K Jolly, S Ahsan

The Royal Shrewsbury and Telford Hospitals NHS Trust
The Shrewsbury and Telford Hospital NHS Trust

\section{Background:}

- The NCEPOD study was commissioned in 2014 (1).

- Aimed to identify difficulties in pathway of care for patients with a stomas in various hospital settings.

- Study highlighted broader issues impacting care of complex patients - For example raised BMI.

- There were several key findings including:

1. Tube obstruction/ displacement $=$ significant morbidity and mortality in ITU.

2. $29.6 \%$ of tracheostomy patients are obese.

3. Adjustable flange tubes are used in $18.8 \%$ of obese patients.

4. $50 \%$ of unplanned tube changes occur patients with a $\mathrm{BMI}>30$

5. Obese patients are at higher risk of complications.

- Poor knowledge in emergency management of stoma patients and a poor awareness of guidance $=$ patient safety issue.

- Operative documentation is crucial in such patients.

- Adjustable flange tracheostomy tubes are a solution to deep necks in patients with limited extension.

\section{Aims:}

- Audit the documentation of key operative information in this subset of patients.

- Consensus of operative documentation for such patients was reached and implemented regionally in two tertiary trusts and served as our standard.

- Ultimately, aim to improve knowledge and their correct use to improve safety in a high-risk patient group.

\section{Method:}

- Retrospective analysis of all tracheostomies over 3year period, identifying variable flange tubes.

- Dual site contribution (district general hospital).

- Analysis of operative notes and discharge summaries.

- Main parameters assessed (documentation standard):

1. Neck anatomy (extension and thickness).

2. Thyroid isthmus division.

3. Tracheal incision type.

4. Tube type (single vs. double lumen).

5. Flange positioning (tube length).

6. Endoscopic confirmation of tube positioning.

\section{Results:}

- Total number of patients reviewed: 68 .

- Total number of patients with adjustable flange tubes inserted: 6 ( $9 \%$ of all tracheostomies performed).

- Tubes used: Portex, dual lumen tube (UniPerc), cuffed and non-fenestrated.

- Grades of surgeons: SpR only.

- Site contribution: RSH: 2 PRH: 4

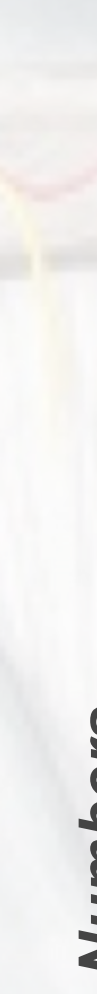

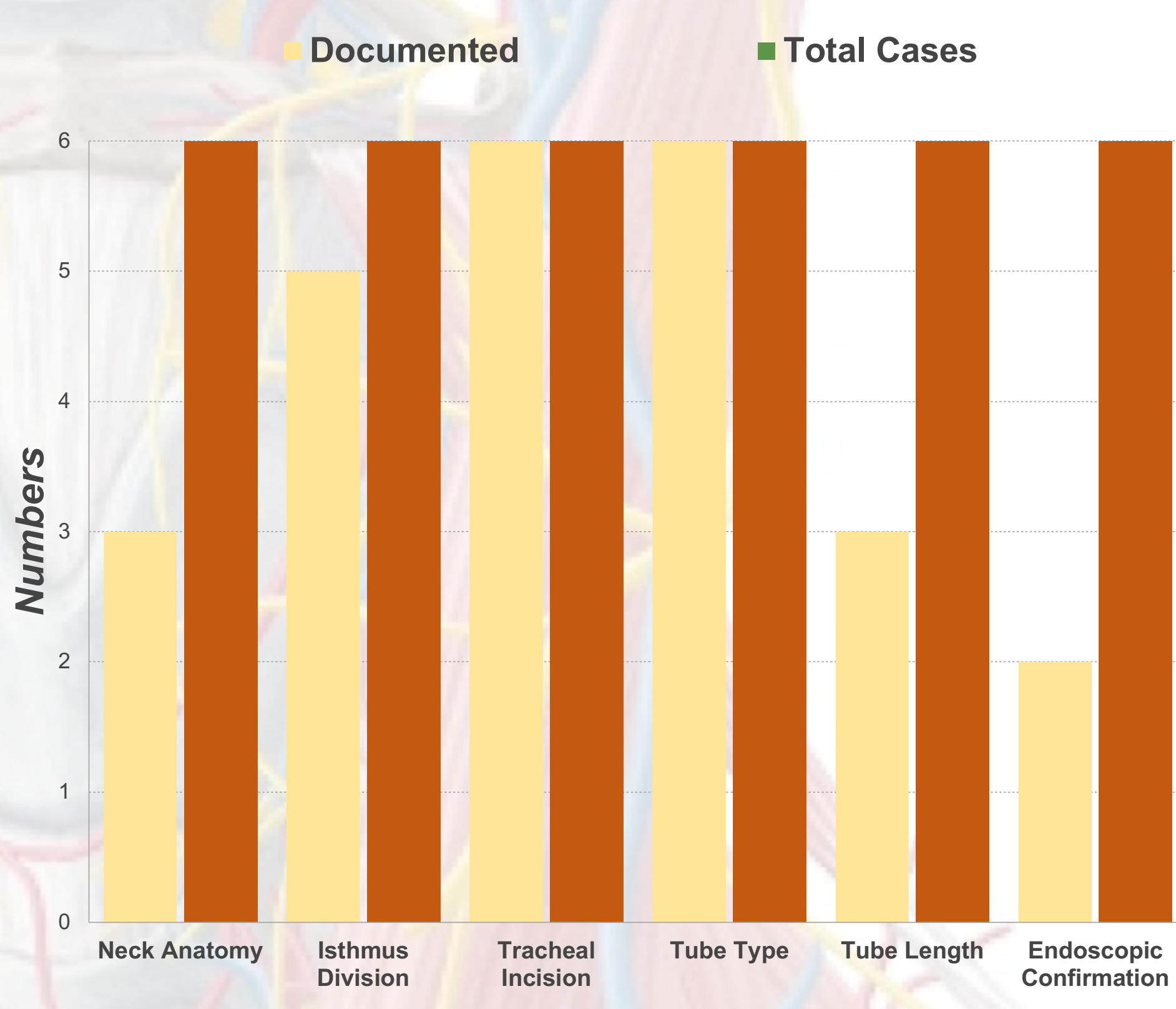

Audit Standard Parameters

\section{Conclusions:}

- Neck stoma related complications remains high despite findings and recommendations from NCEPOD report.

- In the event of displacement, knowledge of intraoperative thyroid isthmus management and tracheal incision is crucial to facilitate tube re-insertion.

- Approximately ten percent of all tracheostomy tubes are variable flange.

- Audit findings demonstrates poor overall documentation throughout trust.

\section{Recommendations:}

- Widespread education regarding the use of adjustable flange tubes and documentation to facilitate healthcare professionals involved in emergency management of stoma patients.

- Introduction of tracheostomy surgical proformas to prevent omission of key informaion.

- Audit data captured across 3 tertiary sites within West Midlands, with ultimate aim being to formulate a national consensus - In conjunction with bodies such as NTSP. 